\title{
O papel da dupla broncodilatação na doença pulmonar obstrutiva crónica: uma revisão baseada na evidência
}

Carla Patrícia Silva, ${ }^{1-2}$ Sara Matos Moreira, ${ }^{1,3}$ Sofia Faria ${ }^{1-2}$

\section{RESUMO}

Objetivo: A doença pulmonar obstrutiva crónica (DPOC) atinge 14,2\% da população portuguesa com mais de 40 anos de idade. Caracteriza-se por uma obstrução do fluxo aéreo, não totalmente reversível, que acarreta dispneia de agravamento progressivo, com impacto nas atividades diárias e na qualidade de vida. As exacerbações estão associadas ao agravamento da doença, ao declínio acelerado da função respiratória e ao aumento da mortalidade.

Esta revisão tem como objetivo determinar, à luz da evidência atual, a eficácia da dupla broncodilatação (anticolinérgico e $\beta$-agonista de longa duração de ação) no tratamento da DPOC, comparativamente com a monoterapia, na melhoria das queixas de dispneia, redução das exacerbações e melhoria da qualidade de vida.

Fontes de dados: MEDLINE, sítios de medicina baseada na evidência e referências bibliográficas dos artigos selecionados.

Métodos de revisão: Foi feita uma pesquisa de normas de orientação clínica, meta-análises, revisões sistemáticas e ensaios clínicos aleatorizados e controlados. Foram utilizados os termos MeSH 'Pulmonary disease, Chronic obstructive', 'Muscarinic antagonists', 'Receptors, Adrenergic, beta-2', 'Drug therapy, Combination' e 'Bronchodilator agents', publicados nos últimos 10 anos, nas línguas inglesa, espanhola e portuguesa.

Resultados: Da pesquisa efetuada obtiveram-se 145 resultados. Destes, foram incluídos um ensaio clínico e quatro normas de orientação clínica. Os restantes foram excluídos por divergirem do objetivo do trabalho ou não cumprirem os critérios de inclusão. $O$ ensaio clínico que compara a dupla broncodilatação (olodaterol e tiotrópio) com o tiotrópio em monoterapia demonstrou o benefício desta associação na função pulmonar, bem como na melhoria da qualidade de vida; porém, não o suficiente para ser considerado clinicamente relevante. As quatro normas de orientação clínica incluídas demonstraram uma melhoria da função pulmonar com a dupla broncodilatação; no entanto, a relevância clínica deste benefício não é clara, nomeadamente ao nível da dispneia, qualidade de vida e taxa de exacerbações.

Conclusões: Da análise dos artigos incluídos há evidência de melhoria da função pulmonar com a dupla broncodilatação comparativamente à monoterapia; no entanto, sem benefício clínico bem estabelecido. Existe ainda necessidade de mais estudos de elevada qualidade, de metodologia homogénea e amostras relevantes que suportem a evidência de melhoria de outcomes orientados para o doente.

Palavras-chave: Doença pulmonar obstrutiva crónica;Antagonistas muscarínicos; Recetores adrenérgicos beta 2; Quimioterapia combinada; Broncodilatadores.

\section{INTRODUÇÃO}

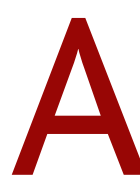
doença pulmonar obstrutiva crónica (DPOC) atinge $14,2 \%$ da população portuguesa com mais de 40 anos de idade. ${ }^{1}$ Caracteriza-se por uma obstrução do fluxo aéreo, não totalmente reversível, que acarreta dispneia de agravamento pro- gressivo, com impacto nas atividades diárias e na qualidade de vida. ${ }^{2-4}$ As exacerbações estão associadas ao

\footnotetext{
1. Médicas Internas de Medicina Geral e Familiar

2. USF Lagoa, Unidade Local de Saúde Matosinhos, EPE

3. USF Leça, Unidade Local de Saúde de Matosinhos, EPE
} 
agravamento da doença, ao declínio acelerado da função respiratória e ao aumento da mortalidade..$^{3-4}$

Os broncodilatadores constituem a pedra angular do tratamento sintomático da DPOC, verificando-se uma melhoria da função pulmonar. No entanto, estes fármacos não demonstraram modificar o declínio da função pulmonar a longo prazo. ${ }^{1}$ As Normas de Orientação Clínica atuais recomendam a administração inalatória de broncodilatadores de longa duração de ação na DPOC moderada a grave..$^{1,3,5-8}$ Os benefícios desta terapêutica incluem não só o controlo sintomático, mas também a melhoria da função pulmonar, da hiperinsuflação, da performance no exercício, do estado de saúde e redução das taxas de exacerbações. ${ }^{9}$

Os broncodilatadores de longa duração de ação, administrados por via inalatória, incluem os $\beta$-agonistas de longa ação (Long-acting $\beta$-agonist - LABA) e os antimuscarínicos de longa ação (Long-acting muscarinic antagonist - LAMA). Nos doentes que, apesar do tratamento de manutenção com LAMA ou LABA em monoterapia, mantêm sintomatologia não controlada está preconizada a associação de broncodilatadores com diferentes mecanismos de ação. A associação LAMA e LABA parece desempenhar um papel importante na maximização da broncodilatação e com bom perfil de tolerância. ${ }^{9-10}$

Na teoria, a associação de LAMA e LABA tem um efeito sinérgico na broncodilatação que advém do relaxamento do músculo liso, de forma direta através da estimulação dos recetores $\beta$-2 adrenérgicos e, indiretamente, por inibição da ação da acetilcolina nos recetores muscarínicos M3. Vários autores têm analisado a fundamentação científica para a aplicabilidade clínica desta dupla broncodilatação na DPOC. ${ }^{11-12}$

Atualmente encontram-se em desenvolvimento diferentes combinações fixas de LAMA e LABA, muitas destas já disponíveis para prescrição. Esta revisão tem como objetivo determinar, à luz da evidência atual, a eficácia da dupla broncodilatação (LAMA e LABA) no tratamento da DPOC, comparativamente com a monoterapia, na melhoria das queixas de dispneia, redução das exacerbações e melhoria da qualidade de vida.

\section{MÉTODOS}

Procedeu-se a uma pesquisa nas bases de dados MEDLINE, National Guidelines Clearinghouse, Guidelines
Finder, Canadian Medical Association Practice Guidelines, Cochrane, DARE, Bandolier e referências bibliográficas dos artigos selecionados, de normas de orientação clínica (NOCs), meta-análises (MA), revisões sistemáticas (RS) e ensaios clínicos aleatorizados e controlados (ECACs). Foram incluídas publicações de janeiro de 2005 a novembro de 2015, nas línguas inglesa, espanhola e portuguesa. Utilizaram-se os termos MeSH 'Pulmonary disease, Chronic obstructive', 'Muscarinic antagonists,' 'Receptors, Adrenergic, beta-2', 'Drug therapy, Combination'e 'Bronchodilatoragents'e os termos DeCS “Doença pulmonar obstrutiva crónica”, "Antagonistas muscarínicos", "Recetores adrenérgicos beta 2", "Quimioterapia combinada" e "Broncodilatadores".

Segundo o modelo PICO, os critérios utilizados para a inclusão dos artigos nesta revisão foram os seguintes: População - doentes com o diagnóstico de DPOC; Intervenção - tratamento inalatório com dupla broncodilatação (LAMA e LABA); Controlo - tratamento inalatório com broncodilatação em monoterapia (LAMA ou LABA); Outcomes - melhoria das queixas de dispneia, redução das exacerbações e melhoria da qualidade de vida.

Para avaliar a qualidade dos estudos e a força de recomendação foi utilizada a escala Strength of recommendation taxonomy (SORT), da American Family Physician. $^{13}$

\section{RESULTADOS}

Na pesquisa inicial foram obtidos 145 resultados. Destes foram excluídos, numa primeira fase, 121 artigos por divergirem do tema desta revisão. Posteriormente foram selecionadas 24 publicações para leitura integral, excluindo-se 19 destas por não cumprirem o objetivo do trabalho, os critérios de inclusão ou por serem repetidas (Figura 1). Assim, foram incluídas cinco publicações: quatro NOCs (Quadro 1) 15-8 $^{5}$ um ECAC (Quadro 2). ${ }^{10}$

A NOC do National Institute for Health and Care Excellence (NICE), de 2010, ${ }^{5}$ afirma que a dupla broncodilatação com formoterol (via inalador de pó seco multidoses) e tiotrópio (via HandiHaler ${ }^{\circledR}$ ), em comparação com o formoterol em monoterapia, tem benefício estatisticamente significativo na função pulmonar, nomeadamente na melhoria do volume expiratório forçado no primeiro segundo (forced expiratory volume in 


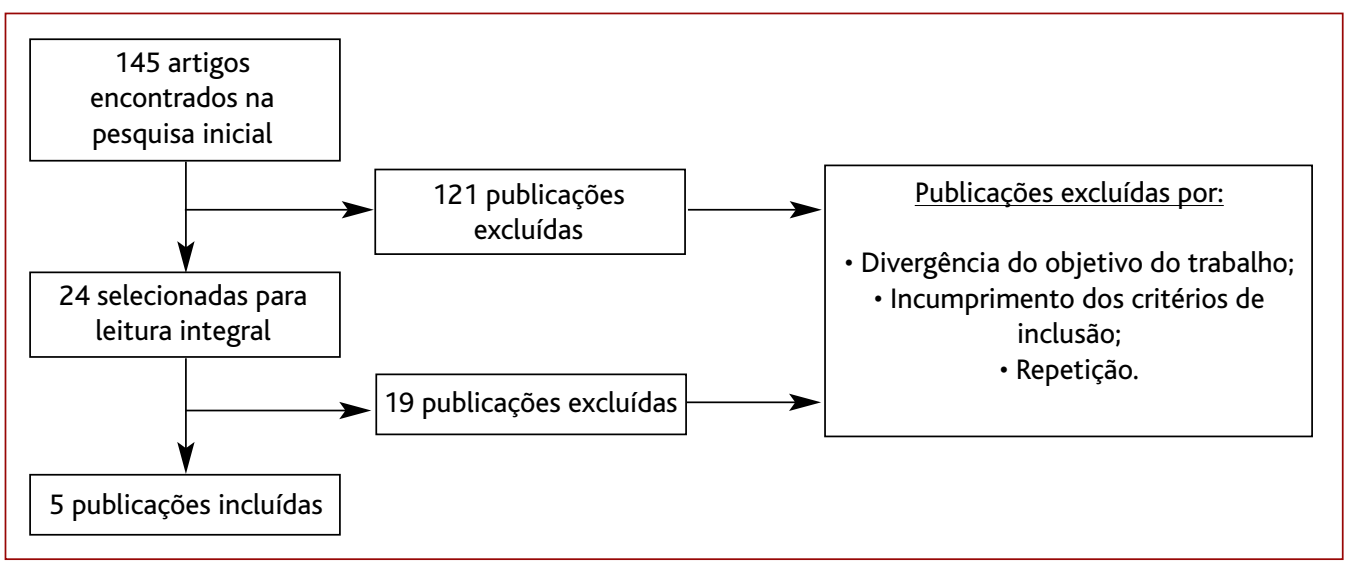

Figura 1. Esquema de seleção de artigos.

\begin{tabular}{|c|c|c|}
\hline $\begin{array}{l}\text { Norma de } \\
\text { Orientação } \\
\text { Clínica }\end{array}$ & Recomendação & $\begin{array}{l}\text { Força de } \\
\text { recomendação }\end{array}$ \\
\hline NICE $(2010)^{5}$ & $\begin{array}{l}\text { A dupla broncodilatação com formoterol e tiotrópio demonstrou benefício na melhoria do } \\
\text { FEV }_{1} \text {. Porém, não se verificou diferença significativa na taxa de exacerbações. }\end{array}$ & $C^{*}$ \\
\hline $\begin{array}{l}\text { NICE } \\
(2014 \mathrm{Feb})^{8}\end{array}$ & $\begin{array}{l}\text { A dupla broncodilatação com glicopirrónio e indacaterol demonstrou melhoria ligeira da } \\
\text { função pulmonar, da dispneia e uso de medicação de alívio sintomático. Porém, a importância } \\
\text { clínica destes benefícios permanece incerta. }\end{array}$ & $C^{*}$ \\
\hline $\begin{array}{l}\text { NICE } \\
(2014 \text { Nov })^{6}\end{array}$ & $\begin{array}{l}\text { A dupla broncodilatação com umeclidínio e vilanterol demonstrou aumento do } \mathrm{FEV}_{1} \text {. No } \\
\text { entanto, a relevância clínica deste benefício não é clara, nomeadamente ao nível da dispneia, } \\
\text { qualidade de vida e taxa de exacerbações. }\end{array}$ & $C^{*}$ \\
\hline $\begin{array}{l}\text { NICE } \\
(2015 \mathrm{Apr})^{7}\end{array}$ & $\begin{array}{l}\text { A dupla broncodilatação com aclidínio e formoterol demonstrou benefícios na melhoria da } \\
\text { função pulmonar, dispneia e taxa de exacerbações, mas apenas clinicamente relevante quando } \\
\text { comparado com placebo. }\end{array}$ & $C^{*}$ \\
\hline
\end{tabular}

Legenda: NICE - National Institute for Health and Care Excellence; *Segundo a Strenght of recommendation taxonomy, da American Family Physician ${ }^{13}$ [atribuído pelos autores].

one second $-\mathrm{FEV}_{1}$ ); porém, não se verifica diferença estatisticamente significativa na taxa de exacerbações. Nesta comparação não há menção quanto ao efeito na melhoria da dispneia nem da qualidade de vida. Quanto à comparação desta dupla broncodilatação com $o$ tiotrópio em monoterapia não há diferença estatisticamente significativa na função pulmonar, na taxa de exacerbações, na dispneia e na mortalidade. Não há referência ao seu papel na qualidade de vida.

Posteriormente, o NICE emitiu três recomendações relativamente a novas associações de broncodilatadores (LAMA e LABA), já disponíveis em Portugal. ${ }^{6-8}$

A associação de glicopirrónio e indacaterol, ${ }^{8} \mathrm{com}$ parativamente ao glicopirrónio ou indacaterol em monoterapia (via Breezhaler ${ }^{\circledR}$, quer na associação quer na monoterapia), bem como na comparação com o tiotrópio (via HandiHaler ${ }^{\circledR}$ ), demonstrou melhoria ligeira mas estatisticamente significativa da função pulmonar (aumento do $\mathrm{FEV}_{1}$ ), da dispneia, do uso de medicação de alívio sintomático e da qualidade de vida. Con- 


\section{QUADRO II. Ensaio clínico}

\begin{tabular}{|c|c|c|c|c|}
\hline Autor e Ano & $\begin{array}{l}\text { Tipo de estudo } \\
\text { e População }\end{array}$ & Metodologia & Resultados & NE \\
\hline $\begin{array}{l}\text { ZuWallack } \\
(2014)^{10}\end{array}$ & $\begin{array}{l}\text { Ensaio aleatorizado } \\
\text { e controlado } \\
\mathrm{N}=2.267 \\
\text { Doentes com } \\
\text { DPOC moderada a } \\
\text { severa }\end{array}$ & $\begin{array}{l}2 \text { Grupos de } \\
\text { tratamento: } \\
\text { (1) olodaterol } 5 \mu \mathrm{g} \\
\text { id e tiotrópio } 18 \mu \mathrm{g} \\
\text { id, via inalatória } \\
\text { (2) tiotrópio } 18 \mu \mathrm{g} \\
\text { id com placebo, via } \\
\text { inalatória }\end{array}$ & $\begin{array}{l}\text { Endpoint primário: } \\
\text { - No Grupo } 1 \text { verificou-se um aumento significativo do } \mathrm{FEV}_{1} \\
(p<0,001) \\
\text { Endpoint secundário: } \\
\text { - No Grupo } 1 \text { verificou-se uma melhoria estatisticamente } \\
\text { significativa da qualidade de vida, com uma diferença de }-1,85 \\
\text { no SGRQ relativamente ao Grupo } 2(-2,757,-0,951 ; \text { IC } 95 \% \text {; } \\
p<0,0001) \text {. No entanto, não é suficiente para ser considerado } \\
\text { clinicamente relevante. }\end{array}$ & 1* \\
\hline
\end{tabular}

Legenda: NE - Nível de evidência; *Segundo a Strenght of recommendation taxonomy, da American Family Physician ${ }^{13}$ [atribuído pelos autores].

tudo, a importância clínica destes benefícios ainda permanece incerta. Esta associação, comparada com o glicopirrónio isoladamente, também demonstrou uma redução estatisticamente significativa da taxa de exacerbações moderadas a graves em doentes com DPOC severa. No entanto, a Agência Europeia do Medicamento (EMA) considera que não é suficiente para suportar a indicação desta associação para reduzir a taxa de exacerbações.

Quanto à associação de umeclidínio e vilanterol, ${ }^{6}$ quando comparada com o vilanterol em monoterapia (via Ellipta®, quer na associação quer na monoterapia), bem como na comparação com o tiotrópio (via HandiHaler $(\AA)$, verificou-se um aumento estatisticamente significativo do $\mathrm{FEV}_{i}$; porém, o mesmo não se verificou na comparação com o umeclidínio em monoterapia. Não obstante, a relevância clínica deste benefício não é clara, sendo a evidência limitada para outcomes orientados para o doente, especificamente a melhoria da dispneia, da qualidade de vida e a redução da taxa de exacerbações. Os estudos não demonstraram diferença estatisticamente significativa na redução da taxa de exacerbações e da dispneia (avaliada segundo o TDI Transition Dyspnoea Index) entre esta associação e o tiotrópio, o umeclidínio ou o vilanterol isoladamente. Na avaliação da qualidade de vida verificou-se uma melhoria, segundo o questionário SGRQ (St. George's Respiratory Questionnaire); porém, a diferença encontra- da foi inferior à necessária para ser considerada clinicamente relevante.

No que se refere à associação de aclidínio e formoterol, ${ }^{7}$ esta demonstrou benefícios na melhoria da função pulmonar, com um aumento estatisticamente significativo do $\mathrm{FEV}_{1}$ uma hora após a inalação, comparativamente com o aclidínio ou formoterol em monoterapia (via Genuair ${ }^{\circledR}$, quer na associação quer na monoterapia) e o placebo. No entanto, após 24 semanas de tratamento, o aumento do $\mathrm{FEV}_{1}$ apenas foi estatisticamente significativo em comparação com o formoterol e o placebo e não com o aclidínio. Esta associação demonstrou de facto uma melhoria estatisticamente significativa da dispneia, avaliada segundo o TDI, quando comparada com a monoterapia. Contudo, verificou-se apenas um aumento inferior a uma unidade no TDI, valor a partir do qual é considerado clinicamente relevante. Na avaliação da qualidade de vida, segundo o questionário SGRQ, a diferença entre a associação e o placebo foi estatisticamente significativa; o mesmo não se verifica quando comparada a associação a cada um dos fármacos. Obteve-se um resultado semelhante no que respeita à taxa de exacerbações.

O ECAC incluído ${ }^{10}$ comparou a dupla broncodilatação com olodaterol (via Respimat ${ }^{\circledR}$ ) e tiotrópio (via HandiHaler ${ }^{\circledR}$ ) (Grupo 1) com o tiotrópio com placebo (via Respimat ${ }^{\circledR}$ ) (Grupo 2) em 2.267 doentes com DPOC moderada a severa. Este estudo avaliou a função pul- 
monar (endpoint primário), bem como o impacto na qualidade de vida, através do questionário SGRQ (endpoint secundário). Contudo, não há referência do seu efeito na taxa de exacerbações nem na melhoria da dispneia. No Grupo 1 verificou-se um aumento significativo do $\mathrm{FEV}_{1}(p<0,001)$ e uma melhoria estatisticamente significativa da qualidade de vida, com uma diferença de -1,85 no SGRQ relativamente ao Grupo 2 (-2,757, $-0,951$; IC 95\%; $p<0,0001)$. No entanto, este resultado não é suficiente para ser considerado clinicamente relevante, uma vez que não atingiu a diferença mínima estabelecida de quatro unidades neste questionário. Este ECAC incluiu dois estudos multicêntricos, randomizados, duplamente cegos.

\section{CONCLUSÃO}

Os estudos incluídos nesta revisão apresentam alguma heterogeneidade relativamente aos fármacos comparados, uma vez que de estudo para estudo varia a associação LAMA e LABA, assim como o LAMA ou LABA do grupo controlo (monoterapia). Além disso, os dispositivos inalatórios diferem, consoante o fármaco utilizado, bem como, em duas das referências estudadas, ${ }^{5,10}$ utilizaram dois dispositivos diferentes para administrar os fármacos em associação, o que pode condicionar a compliance do doente e, assim, comprometer a eficácia do tratamento. Outra limitação é o facto de a dupla broncodilatação em associação fixa ser uma modalidade terapêutica relativamente recente, existindo ainda poucos estudos com outcomes orientados para o doente que suscitem resultados estatisticamente significativos e clinicamente relevantes, não baseados apenas em surrogate endpoints (como parâmetros de função pulmonar como o $\mathrm{FEV}_{1}$ ).

Ainda assim, as NOCs e o ECAC são unânimes quanto ao benefício da dupla broncodilatação na melhoria da função pulmonar. No entanto, a relevância clínica deste benefício não é clara, sobretudo nos outcomes orientados para o doente, nomeadamente na dispneia, qualidade de vida e redução da taxa de exacerbações. Relativamente às NOCs, e apesar de a NICE não atribuir uma força de recomendação a este corpo de evidências, talvez pelos endpoints estudados serem heterogéneos, uns centrados no doente e outros na doença, os autores, de acordo com a Strenght of recommendation taxonomy, da American Family Physician (SORT), atri- buíram a estas NOCs uma força de recomendação C.

Embora os estudos envolvidos apresentem uma boa qualidade metodológica, os resultados estatisticamente significativos basearam-se sobretudo em surrogate endpoints $\left(\mathrm{FEV}_{1}\right)$. Nos outcomes clínicos, como a melhoria das queixas de dispneia, redução das exacerbações e melhoria da qualidade de vida, o uso de dupla broncodilatação não demonstrou benefício claro em comparação com a monoterapia. Embora seja fundamental a existência de evidência sustentada em surrogate endpoints, a prática clínica em medicina geral e familiar deve ser também guiada por outcomes orientados para o doente. Desta forma, esta revisão permite concluir que o papel da dupla broncodilatação permanece por esclarecer relativamente a estes outcomes, sendo que, até à data, apenas o seu benefício nos parâmetros da função pulmonar está comprovado.

Os trabalhos incluídos decorreram maioritariamente em países desenvolvidos, uma realidade provavelmente comparável à portuguesa. À luz do exposto, são ainda necessários mais estudos de elevada qualidade, de metodologia homogénea e amostras relevantes que suportem a evidência de melhoria de outcomes orientados para o doente.

\section{REFERÊNCIAS BIBLIOGRÁFICAS}

1. Direção-Geral da Saúde. Diagnóstico e tratamento da doença pulmonar obstrutiva crónica: norma da DGS n²8/2011, de 30/09/2011. Lisboa: DGS; 2013.

2. U.S. National Library of Medicine. Welcome to medical subject headings! [Internet]. Bethesda, MD: National Institutes of Health; 1999 [updated 2015]. Available from: https://www.nlm.nih.gov/mesh/

3. Global Initiative for Chronic Obstructive Lung Disease. Global Strategy for the diagnosis, management, and prevention of chronic obstructive pulmonary disease [Internet]. GoldCOLD; 2015 [updated 2016]. Available from: http://goldcopd.org/gold-reports/

4. Criner GJ, Bourbeau J, Diekemper RL, Ouellette DR, Goodridge D, Hernandez $\mathrm{P}$, et al. Prevention of acute exacerbations of COPD: American College of Chest Physicians and Canadian Thoracic Society Guideline. Chest. 2015;147(4):894-942.

5. National Institute for Health and Care Excellence. Chronic obstructive pulmonary disease management of chronic obstructive pulmonary disease in adults in primary and secondary care [Internet]. London: NICE; 2010. Available from: https://www.ncbi.nlm.nih.gov/pubmedhealth/ PMH0033903/

6. National Institute for Health and Care Excellence. Chronic obstructive pulmonary disease: umeclidinium/vilanterol combination inhaler (Anoro Ellipta) [Internet]. London: NICE; 2014. Available from: https://www.nice.org.uk/advice/esnm49/chapter/key-points-from- 
the-evidence

7. National Institute for Health and Care Excellence. Chronic obstructive pulmonary disease: aclidinium/formoterol [Internet]. London: NICE; 2015. Available from: https://www.nice.org.uk/advice/esnm57/ chapter/key-points-from-the-evidence

8. National Institute of Health and Care Excellence. Chronic obstructive pulmonary disease: indacaterol/glycopyrronium (Ultibro Breezhaler) [Internet]. London: NICE; 2014.Available from: https://www.nice.org.uk/ advice/esnm33/chapter/key-points-from-the-evidence

9. Beier J, van Noord J, Deans A, Brooks J, Maden C, Baggen S, et al. Safety and efficacy of dual therapy with GSK233705 and salmeterol versus monotherapy with salmeterol, tiotropium, or placebo in a crossover pilot study in partially reversible COPD patients. Int J Chron Obstruct Pulmon Dis. 2012;7:153-64.

10. ZuWallack R, Allen L, Hernandez G, Ting N, Abrahams R. Efficacy and safety of combining olodaterol Respimat $\left({ }^{\circledR}\right)$ and tiotropium HandiHaler $\left({ }^{\circledR}\right)$ in patients with COPD: results of two randomized, doubleblind, active-controlled studies. Int J Chron Obstruct Pulmon Dis. 2014;9:1133-44.

11. Cazzola M, Molimard M. The scientific rationale for combining longacting beta2-agonists and muscarinic antagonists in COPD. Pulm Pharmacol Ther. 2010;23(4):257-67.

12. Lainé DI. Long-acting muscarinic antagonists for the treatment of chronic obstructive pulmonary disease. Expert Rev Clin Pharmacol. 2010;3(1):43-53.

13. Ebell MH, Siwek J, Weiss BD, Woolf SH, Susman J, Ewigman B, et al. Strength of recommendation taxonomy (SORT): a patient-centered approach to grading evidence in the medical literature. Am Fam Physician. 2004;69(3):548-56.

\section{CONFLITOS DE INTERESSE}

As autoras declaram não ter conflitos de interesse.

\section{FINANCIAMENTO DO ESTUDO}

As autoras declaram que o trabalho relatado neste manuscrito não foi objeto de qualquer tipo de financiamento externo.

\section{CONFLITO DE INTERESSES}

Os autores declaram não ter conflitos de interesses.

\section{ENDEREÇO PARA CORRESPONDÊNCIA}

E-mail: carlapbsilva@gmail.com

\section{Recebido em 01-04-2016}

Aceite para publicação em 21-11-2016 


\section{ABSTRACT}

THE ROLE OF DUAL BRONCHODILATION IN CHRONIC OBSTRUCTIVE PULMONARY DISEASE:

\section{AN EVIDENCE-BASED REVIEW}

Objective: Chronic obstructive pulmonary disease (COPD) affects $14.2 \%$ of the Portuguese population over 40 years of age. COPD is characterized by persistent airflow obstruction that is usually progressive, with significant impact on daily activities and quality of life. Exacerbations are associated with an accelerated decline in lung function and an increase in mortality.

This review aims to evaluate the effectiveness of dual bronchodilation using anticholinergic and long-acting $\beta$-agonist drugs in the treatment of COPD, compared with monotherapy, in terms of improvement in dyspnea, reduction of exacerbations, and improved quality of life.

Data sources: MEDLINE, evidence-based medicine websites and bibliographic references of the selected articles were reviewed. Revision methods: A search of clinical guidelines, meta-analyses, systematic reviews and randomized clinical trials, published in the last 10 years, in English, Spanish and Portuguese was performed. The MeSH terms used were 'Pulmonary disease, Chronic obstructive', 'Muscarinic antagonists', 'Receptors, Adrenergic, beta-2', 'Drug therapy, Combination', and 'Bronchodilator agents'.

Results: Of the 145 articles found, five met the inclusion criteria for this review. One article reported the results of a clinical trial and four were guidelines. The clinical trial comparing dual bronchodilation (olodaterol plus tiotropium) with monotherapy with tiotropium found significant improvement in lung function in the first group, as well as improvement in clinical status, but this was not considered to be clinically relevant. The four guidelines claimed improvement in pulmonary function with use of dual bronchodilation. However, the clinical relevance of this benefit was not clear, in terms of reducing dyspnea, improving quality of life, and reducing the number of exacerbations.

Conclusions: Dual bronchodilation improves lung function in patients with COPD, but the clinical benefit of this finding is not clear. Additional studies with similar methodology performed in relevant populations are needed to provide evidence for the beneficial effects of dual bronchodilation on patient-oriented outcomes.

Keywords: Pulmonary disease, Chronic obstructive; Muscarinic antagonists; Receptors, Adrenergic, beta-2; Drug therapy, Combination; Bronchodilator agents. 